\title{
Measuring implementation fidelity in a cluster-randomized pragmatic trial: development and use of a quantitative multi-component approach
}

Miranda B. Olson ${ }^{1,2^{*+}}$ (D), Ellen M. McCreedy ${ }^{1,2,3+}$, Rosa R. Baier ${ }^{1,2,3}$, Renée R. Shield ${ }^{2,3}$, Esme E. Zediker ${ }^{2}$, Rebecca Uth², Kali S. Thomas ${ }^{1,2,3,4}$, Vincent Mor ${ }^{1,2,3,4}$, Roee Gutman ${ }^{3,5}$ and James L. Rudolph 1,3,4

\begin{abstract}
Background: In pragmatic trials, on-site partners, rather than researchers, lead intervention delivery, which may result in implementation variation. There is a need to quantitatively measure this variation. Applying the Framework for Implementation Fidelity (FIF), we develop an approach for measuring variability in site-level implementation fidelity. This approach is then applied to measure site-level fidelity in a cluster-randomized pragmatic trial of Music \& Memory ${ }^{\mathrm{SM}}(\mathrm{M} \& \mathrm{M})$, a personalized music intervention targeting agitated behaviors in residents living with dementia, in US nursing homes (NHs).

Methods: Intervention NHs ( $N=27)$ implemented M\&M using a standardized manual, utilizing provided staff trainings and iPods for participating residents. Quantitative implementation data, including iPod metadata (i.e., song title, duration, number of plays), were collected during baseline, 4-month, and 8-month site visits. Three researchers developed four FIF adherence dimension scores. For Details of Content, we independently reviewed the implementation manual and reached consensus on six core M\&M components. Coverage was the total number of residents exposed to the music at each $\mathrm{NH}$. Frequency was the percent of participating residents in each $\mathrm{NH}$ exposed to M\&M at least weekly. Duration was the median minutes of music received per resident day exposed. Data elements were scaled and summed to generate dimension-level NH scores, which were then summed to create a Composite adherence score. NHs were grouped by tercile (low-, medium-, high-fidelity).
\end{abstract}

\footnotetext{
* Correspondence: miranda_olson@brown.edu

${ }^{\dagger}$ Miranda B. Olson and Ellen M. McCreedy are co-first authors.

${ }^{1}$ Center for Long-Term Care Quality \& Innovation, Brown University School of

Public Health, 121 South Main St., Providence, RI 02912, USA

${ }^{2}$ Center for Gerontology \& Healthcare Research, Brown University School of

Public Health, 121 South Main St., Providence, RI 02912, USA

Full list of author information is available at the end of the article
}

C C The Author(s). 2022 Open Access This article is licensed under a Creative Commons Attribution 4.0 International License, which permits use, sharing, adaptation, distribution and reproduction in any medium or format, as long as you give appropriate credit to the original author(s) and the source, provide a link to the Creative Commons licence, and indicate if changes were made. The images or other third party material in this article are included in the article's Creative Commons licence, unless indicated otherwise in a credit line to the material. If material is not included in the article's Creative Commons licence and your intended use is not permitted by statutory regulation or exceeds the permitted use, you will need to obtain permission directly from the copyright holder. To view a copy of this licence, visit http://creativecommons.org/licenses/by/4.0/ The Creative Commons Public Domain Dedication waiver (http://creativecommons.org/publicdomain/zero/1.0/) applies to the data made available in this article, unless otherwise stated in a credit line to the data. 
Results: The $27 \mathrm{NHs}$ differed in size, resident composition, and publicly reported quality rating. The Composite score demonstrated significant variation across NHs, ranging from 4.0 to 12.0 [8.0, standard deviation (SD) 2.1]. Scaled dimension scores were significantly correlated with the Composite score. However, dimension scores were not highly correlated with each other; for example, the correlation of the Details of Content score with Coverage was $\tau_{\mathrm{b}}=0.11(p=0.59)$ and with Duration was $\tau_{\mathrm{b}}=-0.05(p=0.78)$. The Composite score correlated with CMS quality star rating and presence of an Alzheimer's unit, suggesting face validity.

Conclusions: Guided by the FIF, we developed and used an approach to quantitatively measure overall site-level fidelity in a multi-site pragmatic trial. Future pragmatic trials, particularly in the long-term care environment, may benefit from this approach.

Trial registration: Clinicaltrials.gov NCT03821844. Registered on 30 January 2019, https://clinicaltrials.gov/ct2/show/ NCT03821844.

Keywords: Implementation, Fidelity, Adherence, Pragmatic trial, Nursing home, Dementia

\section{Background}

In recent years, researchers have proposed embedded pragmatic clinical trials (ePCTs) as a potential solution for the persistent gap between efficacy and effectiveness data [1]. While many non-pharmaceutical interventions have proven beneficial in traditional randomized, clinical trials (RCTs), most of these interventions have not been adapted for or tested in real-world settings [1, 2]. ePCT designs minimize researcher involvement in implementation efforts and use pre-existing processes, such as routinely collected data, to capture study outcomes [3]. Unlike traditional RCTs, ePCTs allow for implementation processes and clinical outcomes to be studied simultaneously [4]. This design offers the possibility of accelerating the generation of real-world effectiveness evidence [5].

Because intervention delivery in ePCTs is not led by researchers as in traditional RCTs, capturing variation in implementation is critical for understanding outcome results. One method for capturing this information is to assess implementation fidelity, the degree to which interventions are implemented as intended [6]. Studies show that fidelity is an important potential moderator between intervention delivery and intended outcomes [7-9]. Previous ePCTs have included fidelity analyses [10]. However, many of these trials relied on a simple, exposed versus unexposed quantitative measure for fidelity, such as whether or not a participant viewed the intervention video $[11,12]$. Other studies, applying a more complex definition of implementation fidelity, develop an intervention-specific measurement tool without the guidance of an a priori theoretical framework [13, 14]. Neither of these measurement approaches capture a holistic view of fidelity, especially considering the complexity of many interventions. Other studies, involving a multifaceted definition of implementation fidelity, present mostly qualitative analyses [15]. These analyses remain limited in their usefulness for interpreting the effect of fidelity on quantitative clinical outcomes. There is a need for a method to quantitatively measure variability of implementation fidelity in ePCTs examining complex interventions.

We implemented a cluster-randomized ePCT designed to test the effectiveness of a complex music intervention in the long-term care environment for people living with Alzheimer's Disease and Related Dementias (ADRD). Currently $47 \%$ of United States (US) nursing home (NH) residents live with ADRD, a disease of progressive cognitive dysfunction leading to functional impairments and behavioral changes that require increased supervision over time [16], and the prevalence of Alzheimer's disease is predicted to double by 2060 in the US [17]. Agitated behaviors are common in this population [18] and are often addressed via prescription of psychoactive medications associated with dangerous adverse effects including increased risk of falls and death [19]. With this population of $\mathrm{NH}$ residents rapidly increasing, alternative strategies for managing agitated behaviors are desperately needed.

Personalized music is one alternative strategy which may hold promise to safely manage agitated behaviors in the $\mathrm{NH}$ setting. One popular program, Music and Memory $^{\mathrm{SM}}(\mathrm{M} \& \mathrm{M})$, focuses on using individualized music when a person living with ADRD is prone to agitated behaviors, such as during specific times of day or care actions (e.g., dressing, grooming, bathing) [20]. Research shows that music enjoyed earlier in life may be stored in an area of the brain affected later in the dementia disease course [21]. M\&M leverages the potential of this early preferred music to generate positive emotions in an individual with ADRD, temporarily alleviating agitated behaviors. Many NHs have already adopted M\&M without effectiveness evidence or proven implementation guidance.

Only three evaluations have previously examined the effectiveness of M\&M. So far, there is one relatively 
small trial of this intervention $(N=59)$ [22]. There have also been two larger evaluations of $M \& M$, as administered in a real-world setting. The first is a quasi-experimental study [23], and the second is a pre-post evaluation [24]. These evaluations have resulted in mixed effectiveness evidence regarding the $M \& M$ program, motivating this ePCT. Indeed, the largest $M \& M$ trial to date cites low adherence with high variability in implementation as the rationale for overall null results [22].

As the demand for ePCTs in NHs increases, effectively capturing variation in implementation fidelity is critical for interpreting generated results, particularly in multi-site trials [2]. We implemented an ePCT designed to test the effect of M\&M on agitation among people living with ADRD who reside in geographically and sociodemographically diverse long-term NHs (Clinicaltrials.gov ID: NCT03821844) [25]. This paper presents an approach, guided by a theoretical framework, for measuring sitelevel multi-dimensional implementation adherence to quantitatively describe variability in implementation fidelity across participating US NHs.

\section{Methods}

\section{Study design and participants}

Music \& MEmory: A Pragmatic TRial for Nursing Home Residents With Alzheimer's Disease (METRIcAL) was a parallel, cluster-randomized ePCT of M\&M. This study was designed as a pilot hybrid type 2 trial [26], which simultaneously evaluates intervention effectiveness and implementation $[4,27]$. The details of the trial are described elsewhere (Clinicaltrials.gov ID: NCT03821844) [25]. The trial was conducted in 54 US NHs $(N=27$ intervention, $N=27$ control) from four $\mathrm{NH}$ corporations that differed in size, geographical location, residents' racial composition, and organizational structure. Potentially eligible $\mathrm{NHs}$ had at least 20 residents who were long-stay ( 90 of the last 100 days spent in the $\mathrm{NH}$ ), had a dementia diagnosis, and were not completely deaf. Within each corporation, researchers used $\mathrm{NH}$ characteristics data to identify potentially eligible NHs. Corporate leads were then allowed to exclude NHs known to have pre-existing challenges that may prevent participation, such as recent poor performance surveys or unstable leadership. Depending on the corporation, either researchers or corporate leadership offered the selected NHs the opportunity to participate in the study. Administrators at NHs which decided to opt-in signed a letter of intent describing participation and randomization requirements prior to study initiation. At each intervention facility, the personalized music intervention was implemented via an embedded approach, using a team approach led by a designated NH champion. Recruitment and randomization were completed in February
2019 with the trial running from June 2019 through January 2020. This reporting of the study implementation follows the Standards for Reporting Implementation Studies (StaRI) guidelines as well as the Template for Intervention Description and Replication (TIDieR) guidelines (see Additional File 1). This was deemed a minimal risk study by the Institutional Review Board, which issued a waiver of individual consent (\#1705001793).

\section{Framework for Implementation Fidelity}

Three investigators (MO, EM, and JR) applied the Framework for Implementation Fidelity (FIF) to describe adherence as the primary measurement of implementation fidelity (see Fig. 1) [28]. The FIF presents four dimensions of adherence: (1) Details of Content-what was delivered to participating residents in each $\mathrm{NH}$; (2) Coverage-how many residents received the intervention; (3) Frequency-how often the intervention was delivered to these residents; and (4) Duration-how long the intervention was delivered in each interaction [28]. We apply these four adherence dimensions to our pragmatic trial of M\&M in NHs to qualify and quantify fidelity by aligning with best-practice implementation protocols.

\section{Description of materials}

In the $M \& M$ program, each resident is assigned a personal music device (e.g., iPod) and earphones. NH staff identified music preferred by the resident as a young adult and then loaded this preferred music onto the resident's device. NH staff then initiated music use with the resident at times of day when agitated behaviors are likely or at early signs of agitation [4]. The recommended dose is $30 \mathrm{~min}$ of music per day with each resident.

To promote consistency in intervention delivery and scale-up across the participating NHs, a guide for M\&M implementation was developed during the pilot phase of this study. This implementation guide provides participating NHs with concise, step-by-step guidance on key aspects of the M\&M program.

Participating NHs received two types of staff training: (1) standard M\&M Certification Training and (2) an inperson training developed specifically for this trial. The standard M\&M Certification Training consists of two 90-min live webinars focused on teaching staff how to pilot and scale-up the M\&M program in their $\mathrm{NH}$ [29]. As a supplement to this standard training, corporate leadership and study consultants administered an inperson, study-specific training. This in-person training provided a detailed walk-through of the M\&M implementation guide and allowed for thorough discussion and Q\&A sessions, shared learning between staff, and expanded implementation suggestions, particularly 


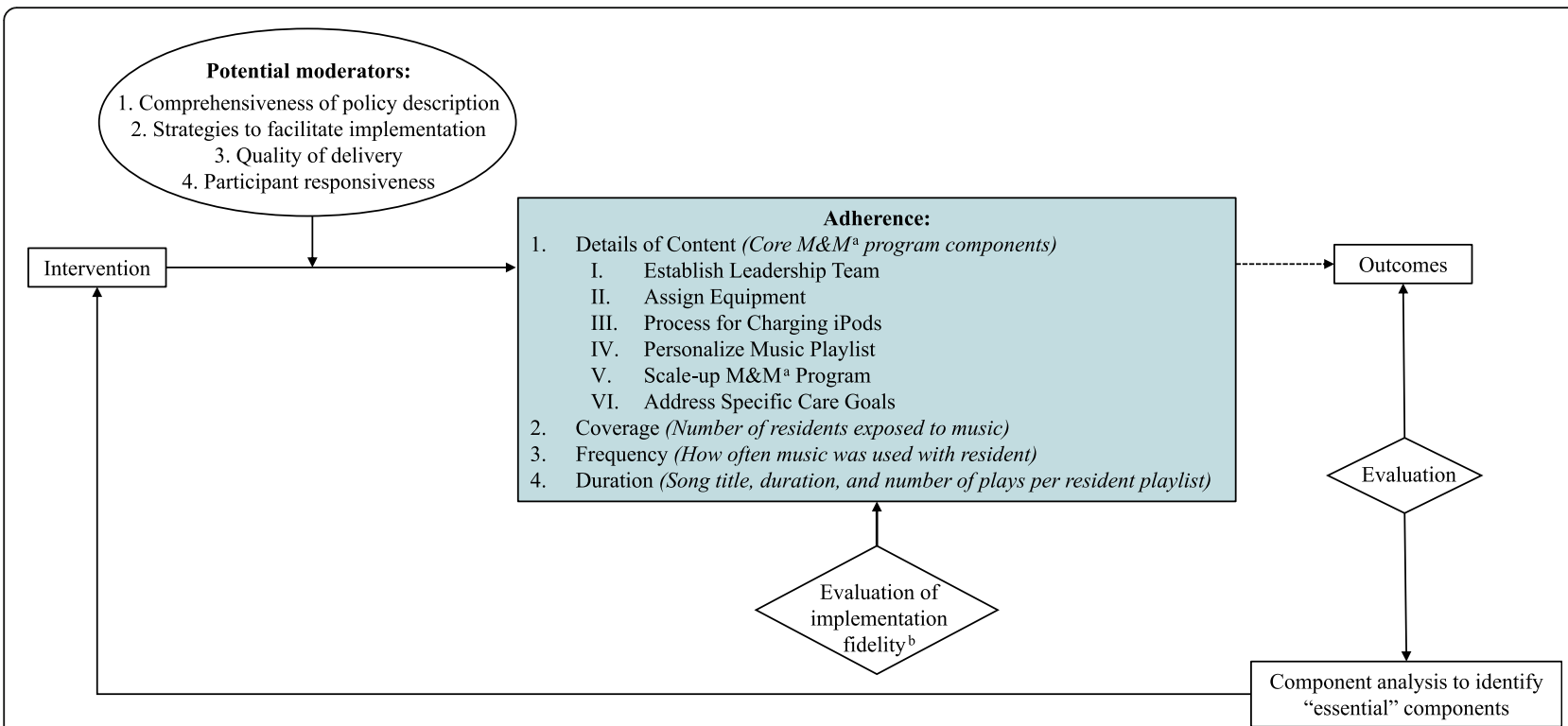

Fig. 1 An overview of the conceptual Framework for Implementation Fidelity as applied to the METRIcAL study. For each adherence dimension presented in the original model, the study-specific definition and identified data elements are summarized

regarding the process for developing an individualized music playlist. The following staff from each $\mathrm{NH}$ were encouraged to attend these in-person sessions: $\mathrm{NH}$ administrator, director of nursing, activities director, social work director, and a nurse manager. For each $\mathrm{NH}$, one attendee was identified as the M\&M champion. In addition to these preparatory trainings, corporate leadership and investigators co-led monthly coaching calls with each $\mathrm{NH}$ to report progress, discuss challenges, and celebrate achievements for the duration of the study.

After completing these staff trainings, each participating $\mathrm{NH}$ was provided with 20 personal music players, 20 charging cables, iTunes gift cards, two multi-port charging stations, three small speakers, and one computer tablet with the iTunes application downloaded.

\section{Data sources}

Several sources of implementation data were collected during the 8-month trial, including (1) interviews with $\mathrm{NH}$ staff, (2) observations by trained data collectors during site visits, (3) metadata downloaded from the music players, (4) forms completed by NH staff at time of first music use, (5) research staff impressions of each $\mathrm{NH}$, and (6) $\mathrm{NH}$ characteristics data from the publiclyavailable Brown University website LTCFocus.org [30].

\section{NH staff interviews}

Ten trained data collectors visited each participating $\mathrm{NH}$ three times during the course of the trial: at baseline, 4 months (mid-implementation), and 8 months (full-implementation). Site visits were conducted over 2 or 3 days and included interviews with $\mathrm{NH}$ staff about each resident's experience while listening to music.

\section{Observation tool}

Following each site visit, the same data collectors completed a structured summary observation form describing how engaged the site was in the M\&M program and different logistical aspects of setup, including individualized labeling of devices and device charging procedures.

\section{iPod metadata}

Using iTunes interface, trained data collectors assisted $\mathrm{NH}$ staff in downloading music play data for each resident. Music play data included the name and artist for every song on the resident's playlist, as well as the duration of each song and the number of times it was played.

\section{Initial Use Form}

For each resident exposed to the program, staff completed an Initial Use Form. This form was completed by the M\&M champion at each NH. The Initial Use Form describes why the resident was chosen for the music program, how and when the resident's personalized music was identified, and how the resident reacted to the music.

\section{Research staff ratings}

Two research assistants who worked closely with $\mathrm{NH}$ staff throughout the study independently scored the engagement of the administrator, nursing staff, and program champion at each $\mathrm{NH}$ as low (1), medium (2), 
or high (3) engagement. The research assistants then met to discuss each score. Consensus was reached, producing three engagement scores for each $\mathrm{NH}$.

\section{LTCFocus.org}

Partially funded by the National Institute on Aging (1P01AG027296), LTCFocus.org is a website and dataset hosted by the Shaping Long Term Care in America Project at Brown University [30]. This source incorporates data from multiple datasets, including the Minimum Data Set (MDS), Certification and Survey Provider Enhanced Reporting (CASPER) system, area resource file, and residential history file. LTCFocus.org was used to capture $\mathrm{NH}$ characteristics in advance of and throughout the study period.

\section{Dimensions of adherence Details of Content}

Capturing what was delivered to residents, Details of Content is the most complex and intervention-specific dimension. In a complex intervention, such as $M \& M$, many components combine to form the intended intervention. However, only some of these components are essential to the primary function of the intervention. These core components may be thought of as the "active ingredients" of the intervention and are actions that $\mathrm{NHs}$ must complete to provide the M\&M intervention, as intended by the intervention's creators [28].

To identify the core components of the M\&M program, three investigators (MO, EM, and JR) first independently reviewed the M\&M implementation manual and then met to discuss the core components each investigator had identified. This process was repeated until the investigators reached consensus, with at least two of the three investigators agreeing on the core components. Two study consultants reviewed and, without suggesting significant edits, approved the final list of $M \& M$ core components. Through this process, we identified six core components of the M\&M program (Table 2).

I. Establish Leadership Team was operationalized as the perceived engagement of $\mathrm{NH}$ staff in M\&M implementation, as engagement seemed to be associated with on-site leadership. The three engagement scores from the Research Staff Ratings were summed for each NH to create a facility-level engagement score.

II. Assign Equipment was operationalized as whether the $M \& M$ equipment was properly labeled for individual residents. The representative data element was the Observational Tool question, "Was each iPod and headphone assigned to an individual resident?" If the data collector observed each iPod and headphone set labeled for an individual resident, the $\mathrm{NH}$ receives a high (1) score. If not all of the equipment was labeled properly, the $\mathrm{NH}$ receives a low (0) score.
III. Process for Charging iPods was operationalized as whether the data collector observed a process for charging equipment at either the midpoint or final data collection. The representative data element was the Observational Tool question, "Did you observe processes for charging iPods?" If the data collector observed processes, the $\mathrm{NH}$ receives a high (1) score. If no processes were observed, the $\mathrm{NH}$ receives a low (0) score.

IV. Personalize Music Playlist was operationalized as the average percent of unique songs on each resident's playlist in a given $\mathrm{NH}$. Using the iPod metadata, the song titles from each resident's playlist were compared to the song titles from the other resident playlists in that $\mathrm{NH}$. The songs not found on the other playlists were considered unique to that resident. The percent of unique songs on each playlist were then averaged for each $\mathrm{NH}$ to generate a facility-level measure.

V. Scale-up MEM Program was operationalized as the difference between the number of residents who were exposed at the midpoint and final data collection visits, compared to the expected numbers at these two timepoints, as established by the M\&M implementation guide (eight and 15 residents, respectively). The Initial Use Form was used to identify each resident's initial date of exposure. For each $\mathrm{NH}$, the number of residents exposed to M\&M at midpoint and final data collection were subtracted from the respective expected number of residents. The absolute values for these differences were then summed to generate a facility-level score.

VI. Address Specific Care Goals was operationalized as the percent of residents in a given $\mathrm{NH}$ who were selected for the program to address a specific goal of care. The Initial Use Form indicates whether a resident was selected for M\&M to address any of the following clinical care goals: acute illness, verbal and physical agitation, bathing, eating, administration of medications, reduction of medications, morning care, pain, and rejection of care. For each $\mathrm{NH}$, the percentage of residents participating in the trial who were selected to address any of these clinical goals was calculated.

\section{Coverage}

Coverage is defined as the number of residents exposed to the intervention. In this study, completion of an Initial Use Form indicates that a specific resident was assigned an iPod and participated in the M\&M program. Therefore, Coverage was calculated as the number of Initial Use Forms completed at each $\mathrm{NH}$ during the study period. The maximum number of participating residents was limited by the number of provided music players.

\section{Frequency}

Frequency is defined as the number of interactions participating residents have with the intervention. However, 
frequency was operationalized as the percentage of participating residents exposed to the music at least once per week by nursing staff. The NH Staff Interviews included the following multiple-choice question: "In the past two weeks, how often have you used the music with the resident?" Based on the response distribution, frequent use of the M\&M program was defined as weekly music use. Therefore, Frequency was calculated as the percentage of participating residents exposed to music at least once per week in each $\mathrm{NH}$, as reported by $\mathrm{NH}$ staff.

\section{Duration}

This was defined as the amount, or length, of intervention provided in each interaction. Duration was operationalized as the median minutes of music received per resident day exposed, averaged over all the residents in a given facility. Using the length of each song and number of plays from the iPod metadata, researchers approximated the total minutes of exposure each resident received during the study period. Exposed days were calculated as the number of days the resident had access to the music, based on the Initial Use Form start date and end of follow-up date. Minutes per exposed day (per resident) is calculated as the total minutes divided by exposed days.

\section{Resident composition and NH characteristics}

Exploratory analyses included several resident composition descriptors including resident gender, race, antipsychotic use, cognitive impairment, and activities of daily living (ADL) score [30]. The following $\mathrm{NH}$ characteristics were also included: number of beds, presence of specialized Alzheimer's unit, Centers for Medicare \& Medicaid Services (CMS) 5-Star Quality Rating [31], payor status, registered nurse hours per resident day, and licensed practical nurse hours per resident day.

\section{Analyses}

To construct a Details of Content score, responses for each $M \& M$ core component were scaled to low (1), medium (2), and high (3) adherence based on data distributions and theoretical significance. These scaled scores were then summed to create an overall Details of Content score. To construct a Composite adherence score, the facility-level score for each FIF adherence dimension was then scaled low (1), medium (2), and high (3) by using the terciles of the corresponding data distributions. These scaled FIF dimension scores were summed to generate a composite adherence score. The raw composite score was then scaled to low (1), medium (2), or high (3) adherence based on the composite score distribution. This scaled Composite score identifies high-, medium-, and low-fidelity NHs.
Fidelity in the FIF dimensions and overall were assessed via descriptive statistics. Kendall's tau-b $\left(\tau_{\mathrm{b}}\right)$ correlations between the six M\&M core components as well as between the FIF dimensions scores and Composite scores are reported. Baseline facility-level characteristics were compared for the high- and low-fidelity $\mathrm{NHs}$, as identified by the Composite adherence score.

\section{Results}

On average, the 27 implementation NHs were large [101.5 beds, standard deviation (SD) 42.3] with primary Medicaid payment source $(58.8 \%$, SD 25.6) and above average quality rating (3.5 Medicare star rating, SD 1.4; range 1-5; higher ratings indicate higher quality) (Table 1). These $\mathrm{NHs}$ on average primarily housed female $(60 \%)$ residents with significant moderate-to-severe cognitive impairment (dementia $=64.1 \%$, SD 11.8) and functional impairment (16.7 Activities of Daily Living score, SD 1.7; range 7-28; higher scores indicate worse function).

Table 1 Characteristics of nursing homes implementing the Music \& Memory program

\begin{tabular}{|c|c|}
\hline & $\begin{array}{l}\text { Intervention Nursing } \\
\text { Homes }(n=27)\end{array}$ \\
\hline & Mean (SD) \\
\hline \multicolumn{2}{|l|}{ Resident composition and acuity ${ }^{a}$} \\
\hline Female (\%) & $65.4(10.9)$ \\
\hline Black (\%) & $22.3(25.7)$ \\
\hline Moderate or severe cognitive impairment (\%) & $64.1(11.8)$ \\
\hline Any antipsychotic use (\%) & $17.9(8.6)$ \\
\hline Total ADL score ${ }^{b}$ & $16.7(1.7)$ \\
\hline \multicolumn{2}{|l|}{ Nursing home characteristics } \\
\hline Total beds (\#) & $101.5(42.3)$ \\
\hline Alzheimer's unit (\%) & $23.1(43.0)$ \\
\hline CMS $^{\complement}$ 5-Star Quality Rating ${ }^{d}$ & $3.5(1.4)$ \\
\hline Medicaid as primary payer (\%) ${ }^{\mathrm{e}}$ & $58.8(25.6)$ \\
\hline Medicare as primary payer (\%) & $11.2(7.0)$ \\
\hline Other/Self-pay (\%) & $30.1(26.4)$ \\
\hline Registered nurse hours per resident day (\#) & $0.3(0.2)$ \\
\hline $\begin{array}{l}\text { Licensed practical nurse hours per resident } \\
\text { day (\#) }\end{array}$ & $0.9(0.3)$ \\
\hline
\end{tabular}

${ }^{a}$ All data represent facility-level characteristics at baseline

${ }^{b}$ Describes ability of resident to perform activities of daily living (ADLs). Higher scores indicate more dependence on staff (range 7-28) [39].

${ }^{\text {C}}$ Centers for Medicare \& Medicaid Services

${ }^{\mathrm{d} S c o r e}$ ranges from 1 to 5 stars, with five stars indicating the highest quality nursing homes [31]

${ }^{\mathrm{e}}$ In the US, Medicare is a health insurance program for citizens aged 65 years and older. Medicare does not provide coverage for long-term care but does provide payment for qualified short-term rehabilitation after hospitalization. Medicaid is a health program for qualified people of all ages related to disability, income, or disease. Medicaid does provide long-term care, and the payment is generally well below that of Medicare. From the nursing home perspective, Medicare is a payor of choice [40] 


\section{Dimensions of implementation fidelity Details of Content}

The Establish Leadership Team score ranges from 3.0 to $9.0(6.4$, SD 2.1), identifying nine low NHs (range 3.0-5.0) and eight high NHs (range 8.0-9.0) for this M\&M component (Table 2). The dichotomized Assign Equipment score (range - 1.0 to 2.0) identifies $7 \mathrm{NHs}$ with a high score (2.0). Similarly, the dichotomized Process for Charging iPods score (range - 2.0 to 2.0) identifies 12 high NHs (range 0.0-2.0). The Personalize Music Playlist score ranges from 0.0 to 1.0 (mean 0.5, SD 0.3) and identifies nine low $\mathrm{NHs}$ (range 0.0-0.1) and nine high NHs (range 0.3-1.0). The Scale-up MEM Program score ranges from 0.0 to 6.0 (mean 2.2, SD 1.7), identifying nine low NHs (range 3.0-6.0) and 11 high NHs (range 0.0-1.0). The Address Specific Care Goals score ranges from 0.0 to 0.9 (mean 0.5, SD 0.3) with nine low NHs (range 0.0-0.3) and nine high NHs (range 0.7-0.9).

The six scaled $M \& M$ core component scores were not highly correlated with one another (Table 3). The Establish Leadership Team and Scale-up MEM Program scores were significantly correlated $\left(\tau_{\mathrm{b}}=0.43, p\right.$ $<0.05)$ as well as the Process for Charging iPods and Address Specific Care Goals scores $\left(\tau_{\mathrm{b}}=0.43, p<\right.$ $0.05)$. The remaining correlations indicate relatively weak associations between the M\&M core components (Table 3).

When summed, the scaled M\&M core component scores generate a Details of Content score ranging from 7.0 to 14.0 (mean 9.6, SD 1.0) with the following tercile ranges: low (7.0-8.0), medium (9.0-10.0), and high (11.0-14.0) (Table 4).

\section{Coverage}

The Coverage score ranges from 4.0 exposed residents to 15.0 (mean 12.4, SD 3.5) (Table 4). The Coverage score identifies nine $\mathrm{NHs}$ in each of the following terciles: low (4.0-13.0), medium (14.0), and high (15.0).

\section{Frequency}

The Frequency score ranges from 0.0 to 1.0 and was normally distributed, with high variability between $\mathrm{NH}$ scores (mean 0.4, SD 0.3) (Table 4). This score identifies nine $\mathrm{NHs}$ in each of the following tercile ranges: low (0.0-0.2), medium (0.3-0.6), and high (0.7-1.0).

\section{Duration}

The Duration score ranges from 0.0 to 99.0 median minutes per resident day exposed (mean 29.8, SD 25.9), with increased playtime indicating the resident received more music (Table 4). This score identifies nine NHs in each of the following tercile ranges: low (0.0-13.0), medium (16.0-28.0), and high (34.0-99.0).

\section{Composite adherence score}

Each scaled FIF dimension score had an equal distribution of NHs across the low (1), medium (2), and high (3) categories (nine NHs per category) (Table 4). These scaled dimension scores were summed to generate a Composite adherence score which ranges from 4.0 to 12.0 (mean 8.0, SD 2.1). This Composite score identifies eight low NHs (4.0-7.0), nine medium NHs (8.0), and ten high NHs (9.0-12.0).

Because the construction of the Composite adherence score is based on the FIF dimensions, the scaled FIF dimension scores were significantly correlated with the Composite score, with the Frequency score displaying the strongest association $\left(\tau_{\mathrm{b}}=0.58, p<0.01\right)$ and the Duration score the weakest association $\left(\tau_{\mathrm{b}}=0.40, p<\right.$ 0.05) (Table 5). However, dimension scores were not highly correlated with each other. For example, the correlation between the Details of Content and the Coverage scores was $\tau_{\mathrm{b}}=0.11(p=0.59)$, and the correlation between the Details of Content and the Duration scores was $\tau_{\mathrm{b}}=-0.05(p=0.78)$ (Table 5$)$. The Frequency score also displays a weak association with the Duration $\left(\tau_{\mathrm{b}}=0.05, p=0.81\right)$ and Coverage $\left(\tau_{\mathrm{b}}=0.14, p=0.42\right)$ scores. The Details of Content and Frequency scores

Table 2 Details of Content: core components of Music \& Memory (M\&M) and corresponding data elements

\begin{tabular}{|c|c|c|c|}
\hline M\&M core component & Data element & Data source & distribution \\
\hline I. Establish Leadership Team & Administrator, Nursing, and Site Champion receptiveness to program & Research Staff Ratings & $\begin{array}{l}\text { Range: } 3.0-9.0 \\
\text { Mean (SD): } 6.4(2.1)\end{array}$ \\
\hline II. Assign Equipment & Was each iPod and headphone assigned to an individual resident? & Observation Tool & $\begin{array}{l}\text { Dichotomized } \\
\text { Frequency: 7, } 20\end{array}$ \\
\hline III. Process for Charging iPods & Did you observe processes for charging iPods? & Observation Tool & $\begin{array}{l}\text { Dichotomized } \\
\text { Frequency: 15, } 12\end{array}$ \\
\hline IV. Personalize Music Playlist & Uniqueness of songs on individual playlists based on song title & iPod Metadata & $\begin{array}{l}\text { Range: } 0.0-1.0 \\
\text { Mean (SD): } 0.5(0.3)\end{array}$ \\
\hline V. Scale-up M\&M Program & Number of residents exposed at midpoint and end of follow-up & Initial Use Form & $\begin{array}{l}\text { Range: } 0.0-6.0 \\
\text { Mean (SD): } 2.2(1.7)\end{array}$ \\
\hline VI. Address Specific Care Goals & Why did you choose this resident to participate in the program? & Initial Use Form & $\begin{array}{l}\text { Range: } 0.0-0.9 \\
\text { Mean (SD): } 0.5(0.3)\end{array}$ \\
\hline
\end{tabular}


Table 3 Correlation coefficients $\left(\tau_{\mathrm{b}}\right)$ and significance values between scaled Music and Memory (M\&M) core component scores

\begin{tabular}{|c|c|c|c|c|c|c|}
\hline & $\begin{array}{l}\text { I. Leadership } \\
\text { team }\end{array}$ & $\begin{array}{l}\text { II. Assign } \\
\text { equipment }\end{array}$ & $\begin{array}{l}\text { III. Charging } \\
\text { iPods }\end{array}$ & $\begin{array}{l}\text { IV. Personalize } \\
\text { playlist }\end{array}$ & $\begin{array}{l}\text { V. Scale-up } \\
\text { M\&M }\end{array}$ & $\begin{array}{l}\text { VI. Specific } \\
\text { care goals }\end{array}$ \\
\hline I. Leadership team & 1.00 & & & & & \\
\hline II. Assign equipment & -0.24 & 1.00 & & & & \\
\hline III. Charging iPods & -0.25 & 0.29 & 1.00 & & & \\
\hline IV. Personalize playlist & 0.23 & -0.21 & 0.11 & 1.00 & & \\
\hline V. Scale-up M\&M & $0.39^{*}$ & -0.24 & 0.05 & -0.11 & 1.00 & \\
\hline VI. Specific care goals & -0.06 & 0.10 & $0.44^{*}$ & -0.11 & 0.21 & 1.00 \\
\hline
\end{tabular}

${ }^{*} p$-value $<0.05$

exhibit the strongest association between the scaled dimension scores $\left(\tau_{\mathrm{b}}=0.43, p<0.05\right)$ (Table 5$)$.

\section{Comparison of high- and low-fidelity NHs}

Using the Composite adherence score, ten high-fidelity NHs and eight low-fidelity NHs were identified (Table 6). On average, high-fidelity $\mathrm{NHs}$ were smaller (mean 89.0 beds, SD 20.7) than the low-fidelity NHs (mean 107.4 beds, SD 28.6) and tended to have a higher percentage of residents with moderate or severe cognitive impairment (65.9\%, SD 16.5\%) than low-fidelity facilities (59.8\%, SD 10.1\%). The high-fidelity NHs had better quality ratings on average (mean 3.6 Medicare star rating, SD 1.4) than the low-fidelity NHs (mean 2.0 Medicare star rating, SD $0.5)$ and were more likely to have an Alzheimer's unit (22.2\%, SD 44.1\%) than the low-fidelity NHs (12.5\%, SD $35.4 \%$ ). Additionally, high-fidelity $\mathrm{NHs}$ have more antipsychotic use on average at baseline (20.6\%, SD 10.1\%) than low-fidelity NHs (16.4\%, SD 10.1\%).

\section{Discussion}

Using the FIF conceptual model, we describe a replicable and feasible approach for quantitatively describing the structure, function, and essential dimensions of implementation fidelity for a cluster-randomized $\mathrm{ePCT}$ of a complex intervention. The four FIF adherence dimension scores-Details of Content, Coverage, Frequency, and Duration-are not highly correlated, highlighting the importance of considering different dimensions of overall implementation fidelity. Initial tests of face validity are promising, as the Composite adherence score is correlated with aspects of $\mathrm{NH}$ quality which are likely to affect implementation including CMS quality star rating and presence of an Alzheimer's specialty unit [15-18]. This study suggests that NHs may perform highly in one adherence domain, but not others. The Composite score successfully captures variability in overall implementation fidelity, indicating that it is feasible and useful to develop a composite measure of implementation fidelity to distinguish facility-level adherence to an intervention implementation protocol. These results have implications for measurement of implementation fidelity in cluster-randomized ePCTs, particularly in the long-term care environment.

Given the findings of previous studies examining the M\&M program [22], METRIcAL focused on thoroughly

Table 4 Framework for Implementation Fidelity (FIF) dimension scores, Composite adherence score, and corresponding data elements

\begin{tabular}{|c|c|c|c|c|c|}
\hline Adherence dimension & Data element(s) & Data source(s) & Construction & Distribution & $\begin{array}{l}\text { High range } \\
\text { NHs }\end{array}$ \\
\hline 1. Details of content & $\begin{array}{l}\text { M\&M core components } \\
\text { data elements }\end{array}$ & Displayed Table 2 & $\begin{array}{l}\text { Data elements were } \\
\text { scaled }(1-3) \text { and } \\
\text { summed }\end{array}$ & $\begin{array}{l}\text { Range: } 7.0-14.0 \\
\text { Mean (SD): } 9.6(1.0)\end{array}$ & $11-14$ (9 NHs) \\
\hline 2. Coverage & $\begin{array}{l}\text { Number of residents } \\
\text { exposed to M\&M program }\end{array}$ & Initial Use Form & $\begin{array}{l}\text { Number of completed } \\
\text { Initial Use Forms }\end{array}$ & $\begin{array}{l}\text { Range: 4.0-15.0 } \\
\text { Mean (SD): } 12.4 \text { (3.5) }\end{array}$ & 15 (9 NHs) \\
\hline 3. Frequency & $\begin{array}{l}\text { In the past } 2 \text { weeks, how } \\
\text { often have you used the } \\
\text { music with the resident? }\end{array}$ & NH Staff Interview & $\begin{array}{l}\text { \% residents in } \mathrm{NH} \text { with } \\
\text { nurses using music at } \\
\text { least once per week }\end{array}$ & $\begin{array}{l}\text { Range: } 0.0-1.0 \\
\text { Mean (SD): } 0.4(0.3)\end{array}$ & $0.67-1.0(9 \mathrm{NHs})$ \\
\hline 4. Duration & $\begin{array}{l}\text { Song title, duration, and } \\
\text { total number of plays per } \\
\text { resident playlist }\end{array}$ & iPod Metadata & $\begin{array}{l}\text { Median minutes of } \\
\text { played music per } \\
\text { resident day exposed }\end{array}$ & $\begin{array}{l}\text { Range: 0.0-99.0 } \\
\text { Mean (SD): } 29.8 \text { (25.9) }\end{array}$ & 30-99 (9 NHs) \\
\hline $\begin{array}{l}\text { Composite } \\
\text { adherence score }\end{array}$ & $\begin{array}{l}\text { Adherence Dimensions } \\
\text { data elements }\end{array}$ & $\begin{array}{l}\text { Adherence Dimensions } \\
\text { Data Sources }\end{array}$ & $\begin{array}{l}\text { Details of content, } \\
\text { Coverage, Frequency, } \\
\text { and Duration data } \\
\text { elements scaled (1-3) } \\
\text { and summed }\end{array}$ & $\begin{array}{l}\text { Range: } 4.0-12.0 \\
\text { Mean (SD): } 8.0(2.1)\end{array}$ & 9-12 (10 NHs) \\
\hline
\end{tabular}


Table 5 Correlation coefficients $\left(\tau_{\mathrm{b}}\right)$ and significance values between scaled FIF dimensions scores and Composite adherence scores

\begin{tabular}{|c|c|c|c|c|c|}
\hline & Details of Content & Coverage & Frequency & Duration & Composite \\
\hline Details of Content & 1.00 & & & & \\
\hline Coverage & $0.10(0.59)$ & 1.00 & & & \\
\hline Frequency & $0.43(0.01)$ & $0.14(0.42)$ & 1.00 & & \\
\hline Duration & $-0.05(0.78)$ & $0.35(0.05)$ & $0.05(0.81)$ & 1.00 & \\
\hline Composite & $0.52(0.00)$ & $0.50(0.00)$ & $0.58(0.00)$ & $0.40(0.02)$ & 1.00 \\
\hline
\end{tabular}

${ }^{*} p$-values presented in parentheses

training participating $\mathrm{NH}$ staff, specifically using the implementation guide tested and revised during the pilot phase of this trial, as well as providing additional support via corporate leads and our research team throughout the study duration. This study identifies six core components essential for achieving complete fidelity, as defined by developers, in implementing the M\&M program. As expected in an ePCT, facility-level variation in implementation fidelity was observed for each core component, despite best efforts to standardize implementation across participating NHs. In general, NHs did not ubiquitously score as high fidelity across the core components. Rather, each $\mathrm{NH}$ tended to have high fidelity for some components and medium or low fidelity for the others. These results highlight the challenges of conducting an ePCT examining a complex intervention. However, the quantitative evaluation of component-level implementation fidelity was feasible because of our rigorous, multimodal data collection approach.

There are precedents for using the FIF as a guiding framework for measuring implementation adherence for this trial design as well as in this care environment [34]. The Pragmatic Trial of Video Education in Nursing Homes (PROVEN), a cluster-randomized ePCT examining the effect of advanced care planning for residents living with ADRD, conducted mixed-methods analyses of implementation fidelity [11]. The PROVEN study presented structured qualitative interview data analyses within the FIF adherence dimensions to contextualize high and low $\mathrm{NH}$ fidelity, as defined by a single

Table 6 Characteristics of low- and high-fidelity nursing homes, as identified by the Composite adherence score

\begin{tabular}{|c|c|c|}
\hline & $\begin{array}{l}\text { Low-fidelity nursing } \\
\text { homes }(n=8)\end{array}$ & $\begin{array}{l}\text { High-fidelity nursing } \\
\text { homes }(n=10)\end{array}$ \\
\hline & Mean (SD) & Mean (SD) \\
\hline \multicolumn{3}{|l|}{ Resident composition and acuity ${ }^{a}$} \\
\hline Female (\%) & $67.5(12.6)$ & $65.0(8.0)$ \\
\hline Black (\%) & $24.7(27.1)$ & $23.1(20.0)$ \\
\hline Moderate or severe cognitive impairment (\%) & $59.8(10.1)$ & $65.9(16.5)$ \\
\hline Any antipsychotic use (\%) & $16.4(10.1)$ & $20.6(10.1)$ \\
\hline Total ADL score ${ }^{b}$ & $16.4(1.4)$ & $17.1(1.9)$ \\
\hline \multicolumn{3}{|l|}{ Nursing home characteristics } \\
\hline Total beds (\#) & $107.4(28.6)$ & $89.0(20.7)$ \\
\hline Alzheimer's unit (\%) & $12.5(35.4)$ & $22.2(44.1)$ \\
\hline CMS $^{\mathrm{C}}$ 5-Star Quality Rating ${ }^{\mathrm{d}}$ & $2.0(0.5)$ & $3.6(1.4)$ \\
\hline Medicaid as primary payer (\%) ${ }^{\mathrm{e}}$ & $63.0(22.2)$ & $67.6(15.2)$ \\
\hline Medicare as primary payer (\%) & $9.6(4.3)$ & $13.8(8.9)$ \\
\hline Other/self-pay (\%) & $27.5(21.6)$ & $18.7(13.8)$ \\
\hline Registered nurse hours per resident day (\#) & $0.3(0.2)$ & $0.3(0.2)$ \\
\hline Licensed practical nurse hours per resident day (\#) & $0.8(0.3)$ & $0.9(0.4)$ \\
\hline
\end{tabular}

${ }^{a}$ All data represent facility-level characteristics at baseline

${ }^{b}$ Describes ability of resident to perform activities of daily living (ADLs). Higher scores indicate more dependence on staff [32].

${ }^{c}$ Centers for Medicare \& Medicaid Services

${ }^{\mathrm{d}}$ Score ranges from 1 to 5 stars, with five stars indicating the highest quality nursing homes [31].

${ }^{\mathrm{e}}$ In the US, Medicare is a health insurance program for citizens aged 65 years and older. Medicare does not provide coverage for long-term care but does provide payment for qualified short-term rehabilitation after hospitalization. Medicaid is a health program for qualified people of all ages related to disability, income, or disease. Medicaid does provide long-term care, and the payment is generally well below that of Medicare. From the nursing home perspective, Medicare is a payor of choice [33] 
quantitative fidelity measure (exposed versus unexposed) [15]. Palmer et al. provided critical lessons learned across the FIF dimensions, but with only one quantitative measure, they did not produce a multi-dimensional quantitative measure of implementation fidelity. However, PROVEN did show that understanding implementation fidelity is critical for properly interpreting the results of pragmatic trials. In the Netherlands, a clusterrandomized ePCT of the Geriatric Care Model, a homebased chronic care management program, also utilized the FIF to measure implementation adherence [35]. Specific questions addressing the four adherence dimensions were collected mid-implementation and after the study concluded, while two moderating factors were assessed after study completion [36]. However, this study of the Geriatric Care Model was limited to qualitative data. Other ePCTs have used the FIF as a guiding framework for implementation fidelity analyses, but none have produced a composite measure of adherence [36-38].

The FIF has also been used as a guiding framework for implementation analyses in RCTs studying complex care interventions in the elderly population $[39,38]$. A notable process evaluation conducted in Norway was the only identified study which constructed a quantitative composite measurement of implementation adherence guided by the FIF [40]. This mixed-methods study accompanied an RCT examining an intervention to promote psychosocial well-being in stroke survivors with and without aphasia during early rehabilitation. This thorough study examines the separate dimensions of implementation adherence, potential modifiers, and presents a quantitative composite measure [40]. While the construction of the composite measure uses a similar approach as the one presented in this paper, Bragstad et al. use theoretically significant cutoff points to define high, medium, and low fidelity. Therefore, the composite measure in this study is used to establish, "that four out of five interventions were implemented with high fidelity," and not to describe variability in overall implementation fidelity [40].

\section{Study limitations}

This study is limited to program implementation in 27 US NHs. Although diverse, this sample of NHs limits the generalizability of the presented results as well as the statistical significance of these analyses. Trained data collectors gathered the included implementation data through interviews, observation, and reporting. Each of these data collection methods present different biases and measurement scales. For example, observer and recall biases may have affected the $\mathrm{NH}$ staff interview responses, particularly as participants and data collectors were not blinded to the intervention. However, through rigorous training and standardized data collection tools, the potential for bias was minimized wherever possible for all data sources. Furthermore, when selecting representative data elements, we retroactively chose data elements that "best fit" the theoretically significant M\&M core components and FIF adherence dimensions. Given the secondary use of some data elements, there may be discrepancy between the intended variable and selected element. Finally, potential moderators included in the FIF conceptual framework (see Fig. 1) likely affect the composite adherence score, but these were beyond the scope of this paper.

\section{Conclusions}

Expanding on previous literature, our team develops and applies an approach to quantitatively measure site-level multi-component implementation adherence for an ePCT using the FIF as a guiding framework. The resulting dimension and Composite adherence scores capture significant variability in implementation fidelity across participating NHs with varied characteristics. By capturing variability in implementation fidelity across the core components of the intervention, the Details of Content dimension allows the presented approach to be adapted for a variety of complex interventions. This approach also allows for differentiation of implementation fidelity across participating sites at various levels: intervention components, dimensions of adherence, and overall fidelity. As indicated by the low association of the dimension scores, these aspects of implementation fidelity are distinct. Therefore, each quantitative sub-score provides a separate opportunity to study the moderating effects of implementation fidelity on primary and secondary clinical outcomes. Additionally, these sub-scores may be used to investigate which intervention components and aspects of adherence are most significant in achieving the desired intervention outcomes. The presented approach may be beneficial for future ePCTs to quantitatively examine the effect of implementation fidelity on study outcomes. Next steps for this work include using the generated dimension and Composite adherence scores to examine the effect of adherence on primary and secondary study outcomes. These analyses will also be extended to include the effect of the FIF potential moderators.

\footnotetext{
Abbreviations

ePCTs: Embedded pragmatic clinical trials; RCTs: Randomized clinical trials; ADRD: Alzheimer's Disease and Related Dementias; US: United States; NHs: Nursing homes; M\&M: Music \& Memory; METRIcAL: Music \& MEmory: A Pragmatic TRial for Nursing Home Residents With Alzheimer's Disease; FIF: Framework for Implementation Fidelity; MDS: Minimum Data Set; CASPER: Certification and Survey Provider Enhanced Reporting; ADL: Activities of Daily Living; CMS: Centers for Medicare \& Medicaid Services; PROVEN: Pragmatic Trial of Video Education in Nursing Homes
} 


\section{Supplementary Information}

The online version contains supplementary material available at https://doi. org/10.1186/s13063-022-06002-8.

Additional file 1. Portable Document Format (.pdf), Reporting Guidelines Checklists. Additional File 1 includes completed checklists for the relevant reporting guidelines - Standards for Reporting Implementation Studies (StaRl) and the Template for Intervention Description and Replication (TIDieR).

\section{Acknowledgements}

We extend great thanks to our consultant collaborators, B\&F Consulting, for lending their expertise throughout this study. We are also exceedingly grateful to the Westat data collectors who went into the field to collect significant aspects of these nursing home data.

\section{Authors' contributions}

Conception or design of the work: MBO, EMM, VM, JLR; acquisition, analysis, or interpretation of data: $M B O, E M M, E E Z, R U, R G, J L R$; and drafted the work or substantively revised it: $M B O, E M M, R R B, R R S, E E Z, R U, K S T, V M, R G$, JLR. All authors read and approved the final manuscript.

\section{Funding}

This research was supported by the National Institutes of Health's National Institute on Aging, grant \#R33AG057451. The sponsor did not have a role in the design, methods, subject recruitment, data collection, analysis, or preparation of paper. This content is solely the responsibility of the authors and does not necessarily represent the official views of the National Institutes of Health's National Institute on Aging. ClinicalTrials.gov identifier: NCT03821844

\section{Availability of data and materials}

The datasets generated and/or analyzed during the current study are available in the Brown Digital Repository, which may be accessed at: https:// doi.org/10.26300/ify9-fa83.

\section{Declarations}

\section{Ethics approval and consent to participate}

The Brown University Institutional Review Board approved this study. The Institutional Review Board deemed this a minimal risk study and issued a waiver of individual consent (\#1705001793).

\section{Consent for publication}

Not applicable

\section{Competing interests}

The authors declare that they have no competing interests.

\section{Author details}

${ }^{1}$ Center for Long-Term Care Quality \& Innovation, Brown University School of Public Health, 121 South Main St., Providence, RI 02912, USA. ${ }^{2}$ Center for Gerontology \& Healthcare Research, Brown University School of Public Health, 121 South Main St., Providence, RI 02912, USA. ${ }^{3}$ Department of Health Services, Policy \& Practice, Brown University School of Public Health, 121 South Main St., Providence, RI 02912, USA. ${ }^{4}$ US Department of Veterans Affairs Medical Center, 830 Chalkstone Ave., Providence, RI 02908, USA. ${ }^{5}$ Department of Biostatistics, Brown University School of Public Health, 121 South Main St., Providence, RI 02912, USA.

Received: 18 August 2021 Accepted: 4 January 2022

Published online: 15 January 2022

\section{References}

1. Weinfurt KP, Hernandez AF, Coronado GD, DeBar LL, Dember LM, Green BB, et al. Pragmatic clinical trials embedded in healthcare systems: generalizable lessons from the NIH Collaboratory. BMC Med Res Methodol. 2017;17(1):144 Available from: https://pubmed.ncbi.nlm.nih.gov/28923013.

2. Gitlin LN, Marx K, Stanley IH. Hodgson N. Translating evidence-based dementia caregiving interventions into practice: state-of-the-science and next steps. Gerontologist. 2015;55(2):210-26 Available from: https://pubmed ncbi.nlm.nih.gov/26035597.

3. Chalkidou K, Tunis S, Whicher D, Fowler R, Zwarenstein M. The role for pragmatic randomized controlled trials ( $p R C T s$ ) in comparative effectiveness research. Clin Trials J Soc Clin Trials. 2012;2, 9(4):-446. https://doi.org/10.11 77/1740774512450097.

4. Curran GM, Bauer M, Mittman B, Pyne JM, Stetler C. Effectivenessimplementation hybrid designs: combining elements of clinical effectiveness and implementation research to enhance public health impact. Med Care. 2012;50(3):217-26. Available from: https://pubmed. ncbi.nlm.nih.gov/22310560. https://doi.org/10.1097/MLR.0b013e31824 08812.

5. Glasgow RE, Magid DJ, Beck A, Ritzwoller D, Estabrooks PA. Practical clinical trials for translating research to practice: design and measurement recommendations. Med Care, 7. 2005;43(6):551 Available from: https://journa Is.lww.com/lww-medicalcare/Fulltext/2005/06000/Practical_Clinical_Trials_ for_Translating_Research.5.aspx.

6. Dusenbury $L$, Brannigan $R$, Falco M, Hansen WB. A review of research on fidelity of implementation: implications for drug abuse prevention in school settings. Health Educ Res. 2003;18(2):-256. https://doi.org/10.1093/her/1 8.2.237.

7. Hill HC, Erickson A. Using implementation fidelity to aid in interpreting program impacts: a brief review. Educ Res. 2019;48(9):590-8. Available from. https://doi.org/10.3102/0013189X19891436.

8. Elliott DS, Mihalic S. Issues in disseminating and replicating effective prevention programs. Prev Sci. 2004;5(1):47-53. Available from. https://doi. org/10.1023/B:PREV.0000013981.28071.52.

9. Inouye SK, Bogardus ST Jr, Williams CS, Leo-Summers L, Agostini JV. The role of adherence on the effectiveness of nonpharmacologic interventions: evidence from the delirium prevention trial. Arch Intern Med. 2003;163(8):958-64. Available from. https://doi.org/10.1001/a rchinte.163.8.958.

10. Gesell SB, Bushnell CD, Jones SB, Coleman SW, Levy SM, Xenakis JG, et al. Implementation of a billable transitional care model for stroke patients: the COMPASS study. BMC Health Serv Res. 2019;19(1):978 Available from: https://pubmed.ncbi.nlm.nih.gov/31856808.

11. Mitchell SL, Volandes AE, Gutman R, Gozalo PL, Ogarek JA, Loomer L, et al. Advance care planning video intervention among long-stay nursing home residents: a pragmatic cluster randomized clinical trial. JAMA Intern Med. 2020;180(8):1070-8. Available from. https://doi.org/10.1001/jamainternmed.2 020.2366

12. Lin S-Y, Schneider CE, Bristol AA, Clancy M, Sprague SA, Aldridge M, et al. Findings of sequential pilot trials of Aliviado Dementia Care to inform an embedded pragmatic clinical trial. Gerontologist. 2020:gnaa220 Available from. https://doi.org/10.1093/geront/gnaa220.

13. Axford N, Bjornstad G, Clarkson S, Ukoumunne OC, Wrigley Z, Matthews J, et al. The effectiveness of the KiVa bullying prevention program in Wales, UK: results from a pragmatic cluster randomized controlled trial. Prev Sci. 2020;21(5):61526. Available from. https://doi.org/10.1007/s11121-020-01103-9.

14. Toomey E, Matthews J, Guerin S, Hurley DA. Development of a feasible implementation fidelity protocol within a complex physical therapy-led self-management intervention. Phys Ther. 2016;96(8):1287-98. Available from. https://doi.org/10.2522/ptj.20150446.

15. Palmer JA, Parker VA, Barre LR, Mor V, Volandes AE, Belanger $E$, et al. Understanding implementation fidelity in a pragmatic randomized clinical trial in the nursing home setting:a mixed-methods examination. Trials. 2019; 20(1):656. Available from. https://doi.org/10.1186/s13063-019-3725-5.

16. Harris-Kojetin L, Sengupta M, Lendon J, Rome V, Valverde R, Caffrey C. Long term care providers and services users in the United States, 2015-2016. Vital Heal Stat. 2019:3(43):23.

17. Matthews KA, Xu W, Gaglioti AH, Holt JB, Croft JB, Mack D, et al. Racial and ethnic estimates of Alzheimer's disease and related dementias in the United States (2015-2060) in adults aged $\geq 65$ years. Alzheimers Dement. 2019;15(1): 17-24 Available from: https://pubmed.ncbi.nlm.nih.gov/30243772.

18. Zuidema S, Koopmans R, Verhey F. Prevalence and predictors of neuropsychiatric symptoms in cognitively impaired nursing home patients. J Geriatr Psychiatry Neurol. 2007;29(1):-49. https://doi.org/10.1177/0891 988706292762

19. Chiu Y, Bero L, Hessol NA, Lexchin J, Harrington C, et al. Health Policy (New York). 2015;119(6):802-13 Available from: https://www.sciencedirect.com/ science/article/pii/S0168851015000652. 
20. Why Get Certified: Music and Memory. https://musicandmemory.org. Accessed 1 January 2021.

21. Jacobsen J-H, Stelzer J, Fritz TH, Chételat G, La Joie R, Turner R. Why musical memory can be preserved in advanced Alzheimer's disease. Brain. 2015; 138(8).

22. Kwak J, Anderson $\mathrm{K}, \mathrm{O}^{\prime}$ Connell VK. Findings from a prospective randomized controlled trial of an individualized music listening program for persons with dementia. J Appl Gerontol. 2020;39(6):-575. https://doi.org/10.1177/ 0733464818778991.

23. Thomas KS, Baier R, Kosar C, Ogarek J, Trepman A, Mor V, et al. Am J Geriatr Psychiatry. 2017;25(9):931-8 Available from: https://pubmed.ncbi.nlm.nih. gov/28483436.

24. Bakerjian D, Bettega K, Cachu AM, Azzis L, Taylor S, et al. J Am Med Dir Assoc. 2020;21(8):1045-1050.e2 Available from: https://pubmed.ncbi.nlm.nih. gov/32146039.

25. McCreedy EM, Yang X, Baier RR, Rudolph JL, Thomas KS, Mor V. Measuring effects of nondrug interventions on behaviors: Music \& Memory pilot study. J Am Geriatr Soc. 2019;67(10):-2138. https://doi. org/10.1111/jgs.16069

26. McCreedy EM, Gutman R, Baier R, Rudolph JL, Thomas KS, Dvorchak F, et al. Measuring the effects of a personalized music intervention on agitated behaviors among nursing home residents with dementia: design features for cluster-randomized adaptive trial. Trials. 2021;22(1):681. Available from. https://doi.org/10.1186/s13063-021-05620-y.

27. Landes SJ, McBain SA, Curran GM. An introduction to effectivenessimplementation hybrid designs. Psychiatry Res. 2019;280:112513 Available from: https://pubmed.ncbi.nlm.nih.gov/31434011.

28. Carroll C, Patterson M, Wood S, Booth A, Rick J, Balain S. A conceptual framework for implementation fidelity. Implement Sci. 2007;2(1):40. Available from. https://doi.org/10.1186/1748-5908-2-40.

29. MUSIC \& MEMORY ${ }^{\circledast}$ Certification Training. https://musicandmemory.org/gettrained/certification-training/. Accessed 1 March 2021.

30. Long-term Care: Facts on Care in the US. Brown University Center for Gerontology and Healthcare Research. 2019. https://tcfocus.org. Accessed 1 January 2020.

31. Five-Star Quality Rating System. Centers for Medicare and Medicaid Services. 2019. https://www.cms.gov/Medicare/Provider-Enrollment-and-Certification/ CertificationandComplianc/FSQRS. Accessed 1 January 2020.

32. Morris JN, Fries BE, Morris SA. Scaling ADLs Within the MDS. J Gerontol Ser A. 1999;54(11):M546-M553. Available from: https:/doi.org/10.1093/gerona/ 54.11.M546

33. Medicare and Medicaid Basics - MLN Booklet. Centers for Medicare and Medicaid Services. 2018. https://www.cms.gov/Outreach-and-Education/ Medicare-Learning-Network-MLN/MLNProducts/Downloads/ProgramBasics. pdf. Accessed 11 December 2021.

34. Hasson H. Systematic evaluation of implementation fidelity of complex interventions in health and social care. Implement Sci. 2010;5(1):67. Available from. https://doi.org/10.1186/1748-5908-5-67.

35. Muntinga ME, Van Leeuwen KM, Schellevis FG, Nijpels G, Jansen APD. From concept to content: assessing the implementation fidelity of a chronic care model for frail, older people who live at home. BMC Health Serv Res. 2015;15(1):18. Available from. https://doi.org/10.1186/ s12913-014-0662-6.

36. Willeboordse F, Schellevis FG, Meulendijk MC, Hugtenburg JG, PJM E. Implementation fidelity of a clinical medication review intervention: process evaluation. Int J Clin Pharm. 2018;40(3):550-65 Available from: https:// pubmed.ncbi.nlm.nih.gov/29556930.

37. Berry CA, Nguyen AM, Cuthel AM, Cleland CM, Siman N, Pham-Singer $H$, et al. Measuring implementation strategy fidelity in HealthyHearts NYC: a complex intervention using practice facilitation in primary care. Am J Med Qual. 2020. Available from;36(4):270-6. https://doi.org/10.1177/106286062 0959450.

38. Smith J, Green J, Siddiqi N, Inouye SK, Collinson M, Farrin A, et al. Investigation of ward fidelity to a multicomponent delirium prevention intervention during a multicentre, pragmatic, cluster randomised, controlled feasibility trial. Age Ageing. 2020;49(4):648-55. Available from. https://doi. org/10.1093/ageing/afaa042.

39. Hasson $\mathrm{H}$, Blomberg S, Dunér A. Fidelity and moderating factors in complex interventions: a case study of a continuum of care program for frail elderly people in health and social care. Implement Sci. 2012;7:23 Available from: https://pubmed.ncbi.nlm.nih.gov/22436121.
40. Bragstad LK, Bronken BA, Sveen U, Hjelle EG, Kitzmüller G, Martinsen R, et al. Implementation fidelity in a complex intervention promoting psychosocial well-being following stroke: an explanatory sequential mixed methods study. BMC Med Res Methodol. 2019;19(1):59 Available from: https://pubmed.ncbi.n/m.nih.gov/30876403.

\section{Publisher's Note}

Springer Nature remains neutral with regard to jurisdictional claims in published maps and institutional affiliations.
Ready to submit your research? Choose BMC and benefit from:

- fast, convenient online submission

- thorough peer review by experienced researchers in your field

- rapid publication on acceptance

- support for research data, including large and complex data types

- gold Open Access which fosters wider collaboration and increased citations

- maximum visibility for your research: over $100 \mathrm{M}$ website views per year

At BMC, research is always in progress.

Learn more biomedcentral.com/submissions 